\title{
PHOTOPOLYMERIZABLE ACRYLIC MIXTURES WITH SPECIAL PROPERTIES FOR OPTICAL DISCS AND MULTILAYER GLASSES PRODUCING
}

\author{
ALFRED ROT and IRENE ZAKS \\ R\&D Center "Fast,Ltd", p.o.Box 5457, Lvov 290031, Ukraine \\ Institute of Wood Chemistry, Latvian Akademy of Sciences, \\ Akademijas st.27, Riga 226006, Latvia
}

The formation of densely cross-linked networks by chain cross-linking photopolymerization is discussed in relation to selected applications in the high technology. All of these applications make use of the speed and of the latitude to meet other requirements by variation of the chemical structure of the monomers. The selection comprises: 1 - the replication of optical discs; 2 - the bonding of laminated glasses.

\section{Introduction}

Photopolymerization is a technique which allows the rapid conversion at room temperature of liquid materials into rubbery or glassy products. It is widely applied in several branches of the industry. Optical discs are manufactured by replication from a mold. Photopolymerization is just one of the available methods for copying discs. It has been used for several years in the large scale production of video discs [1]. In addition photopolymerization serves for making pre-groove structures on Direct Read After Write (DRAW) discs [2].

A critical step in the development of the photoreplication process has been the choice of a proper photopolymerizable coating. The coating must have a high curing rate and a low viscosity. In the cured state, it must have high refractive index and thermostability, low volume shrinkage and birefringence, optimum hardness and tensile strength. Coatings which meet all requirements are not easy to find, largely because some of requirements are more or less conflicting. An example is easy release from the metallic mold and good adhesion to the metallic reflective coating.

For the production of laminated glasses it is known to bond glass to glass by means of a film of an organic polymer such as polyvinylbutyral. However, when such an adhesive is employed, a complicated time-consuming process is required for the lamination. It has been proposed to use 
a photocurable adhesive for glass lamination [3] and various formulations for these adhesives. However, none of them fully satisfies the requirements for the adhesion problems.

Acrylates have been chosen as preferred components of the above mentioned formulations on account of their high rates of polymerization. By chemical modification of ester groups, materials with very different properties may be obtained without sacrifying too much of the polymerization rate. Knowing that there is a wide range of monomers offered to the photocuring industry, we felt the need to help the formulator in selecting the right and modern monomers and oligomers.

On the other hand we were interested to clear the question of how to overcome the problems of adhesion of photocuring systems to non-porous substrates. The shrinkage during photopolymerization in combination with the fact that photocuring systems are most of the time without solvent and the instantaneous cross-linking is so rapid, not giving time to show relaxations is often used as the principal explanation for the difficulties in adhesion. Such correlations were also interesting for us.

\section{Experimental}

\section{Materials}

In order to make it useful to as many formulators as possible, we selected both the widely used and new monomers, which are presented in table 1.

Table 1.

\section{Monomers used for formulations}

\begin{tabular}{|c|c|c|}
\hline No. & Chemical Name & Abbr. \\
\hline & Reactive Diluents & \\
\hline 1 & Isobornyl Acrylate & IBOA \\
\hline 2 & $\begin{array}{l}2 \text { - hydroxy - } 3 \text { - chlorinpropyl } \\
\text { acrylate }\end{array}$ & HCPA \\
\hline 3 & $\begin{array}{l}\text { Tribromoneopentylalcochol Carbonate } \\
\text { Methacrylate }\end{array}$ & TBNPACM \\
\hline 4 & $\begin{array}{l}\text { Allyl Carbonate Methacrylate } \\
\text { Diacrylates of Glycols }\end{array}$ & ALCM \\
\hline 5 & 1.6 - Hexnediol Diacrylate & HDDA \\
\hline 6 & Triethylenglycol Diacrylate & TEGDA \\
\hline 7 & Tripropylenglycol Diacrylate & TPGDA \\
\hline 8 & Tetraethyleneglycol Diacrylate & TrEGDA \\
\hline 9 & Polyethyleneglycol 200 Diacrylate & PEG 200DA \\
\hline 10 & Polyethyleneglycol 400 Diacrylate & PEG 400DA \\
\hline 11 & Propoxylated Neopentylglycol Diacrylate & NPGPODA \\
\hline 12 & Ethoxylated Bisphenol A Diacrylate & BPADA \\
\hline 13 & Ethoxylated Bisphenol A Dimethacrylate & BPADM \\
\hline
\end{tabular}


No. Chemical Name

14

15

16

17

18

19
Tetrabromodipentaerythritol Dicarbonate Methacrylate Dibromoneopentylglycol Dicarbonate Methacrylate

1.4 - Butanediol Diacrylate

Polyfunctional Acrylates

7 Highlyethoxylated Trimethylopropane

Triacrylate

Dipentaerythritol Pentaacrylate

Xylitol Pentacarbonate Methacrylate
Abbr.

TBDPDCM

DBNPGDCM

BDDA

HEOTMPTA

DPETPA

XPCM

We chose for some optical disc's formulations new oligomer EAS-637, a resorcin type epoxy acrylate [4] of the following structure

$\left.\mathrm{CH}_{2}=\mathrm{CHCOOCH}_{2} \mathrm{CHCH}_{2}+\mathrm{O}-\mathrm{OCH}_{2} \mathrm{CHCH}_{2}\right)_{1.11} \mathrm{OCOCH}=\mathrm{CH}_{2}$

and for glasses adhesives three new oligomers (table 2).

Table 2

Oligomers for glasses adhesives

\begin{tabular}{clcl}
\hline Name & M.w. & Functionality & Description \\
\hline IF 491 & low & 3 & $\begin{array}{l}\text { aromatic urethane } \\
\text { methacrylate } \\
\text { IF 591 }\end{array}$ medium \\
IF 691 & high & 2 & $\begin{array}{l}\text { aliphatic polyester } \\
\text { epoxymethacrylate } \\
\text { aromatic polyester urethane } \\
\text { epoxy methacrylate }\end{array}$ \\
\hline
\end{tabular}

We used the oligomers produced in the laboratory for this study.

The photoinitiators used are Irgacure 651, Darocure 1173 (Merck) and 2.2diisopropoxyacetophenone, synthesized in laboratory [5].

\section{Test Methods}

Viscosity was measured with a Brookfild RVF-100 viscometer at $25^{\circ} \mathrm{C}$ and reported in mPa.s.

Cure speed was examined on a $10 \mathrm{~mm}$ draw-down on paper, by passing several times under a $40 \mathrm{~W} / \mathrm{cm}$ UV-lamp DRT-1000. The intensity at $365 \mathrm{~nm}$ was $100 \mathrm{~W} \cdot \mathrm{m}^{-2}$. Reported are the numbers of passages, needed to get a fully cured surface. Fully cured meaning: not marking after 
two subsequent finger-rubs. On the other hand in some cases the rate of polymerization was measured using PERKIN-ELMER photo DSC. The samples contained $2 \mathrm{wt} . \%$ photoinitiator Irgacure 651 were irradiated under nitrogen at $0.2 \mathrm{~mW} / \mathrm{cm}^{2}$.

Shrinkage during polymerization was measured in the interferometer owing probing light with $\lambda=633.1 \mathrm{~nm}$ intensity dependence in the fixed point of the interference pattern upon irradiation time [6].

Refractive index was measured with an Abbe refractometer.

Birefringence index was measured by an elipsometer at $\lambda=780 \mathrm{~nm}$.

Glass transition temperature and temperature of pyrolysis were measured using PERKINELMER DMA and TGA.

Surface hardness was determined by indention of a diamond point under a load with an apparatus PMT-3.

Tensile strength was determined with "Tiratest 2160".

Adhesion was measured with the following method. The coating was applied at a thickness of $30 \mathrm{mkm}$ to the glass substrate. Test cylinders with a diameter of $20 \mathrm{~mm}$ were attached to the coating and the back of the substrate with a two-pack epoxy-adhesive. The test assembly was left for 7 days to allow the adhesive to cure. For testing the assembly is pulled apart using the special tensometer which is a constant strain/variable stress instrument. The result obtained is divided by the surface area and is expressed in $\mathrm{xN} / \mathrm{cm}^{2}$.

\section{Results and discussion}

\section{Cure Speed}

As to be expected, high functionality results in high reactivity. Notice the highest reactivity of all, is found with DPETPA and especially XPCM. Generally speaking, the replacement of (meth) acrylates by the corresponding carbonate (meth)acrylates increases the rate of photopolymerization. The results of the measuring the rate of polymerization of TBDPDCM are shown in Fig.1.

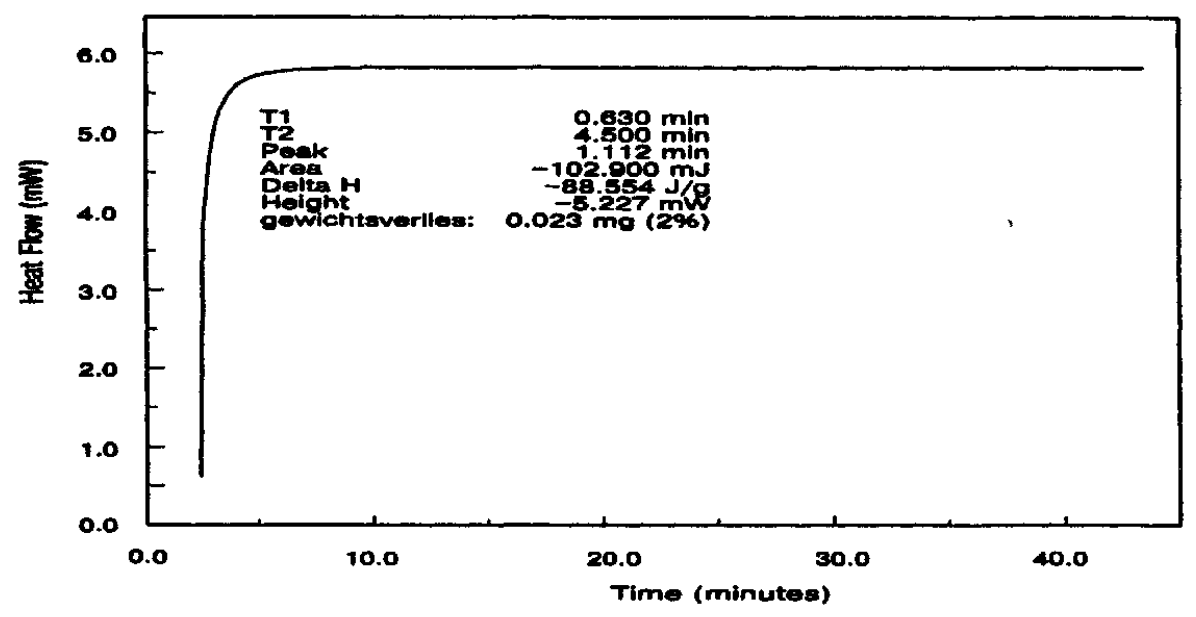

Fig.1. Photo DSC of TBDPDCM 
Notice in this respect the remarkable reactivity found with ALCM. The explanation for this, besides the combination of two different types of the unsaturation, might also be extremely low viscosity, found in this particular case. This enables cross-linking to an ultimate extent.

It was also established that TEGBDA and HDDA showed the optimum combination of the reaction rate and conversion. The preference was given to HDDA due to its higher purity and better availability. All the above mentioned compounds have been applied in the optical discs replication technology $[1,2,7,8]$.

\section{Shrinkage}

The smaller the shrinkage of optical disc's monomers the smaller is the shape correction of the mold. Overall shrinkage may be reduced by increasing the length of the moiety between the acrylate groups, but this occurs at the cost of a reduced cross-link density and hardness. The last disadvantage may be overcome in part by using a dimethacrylate instead of a diacrylate. The best results were achieved by using ALCM, BPADM, TBDPDCM, DBNPGDCM, DPETPA and XPCM. Polymerization shrinkage for BPADM (curve 1) and DBNPGDCM (curve 2) is shown in Fig.2.

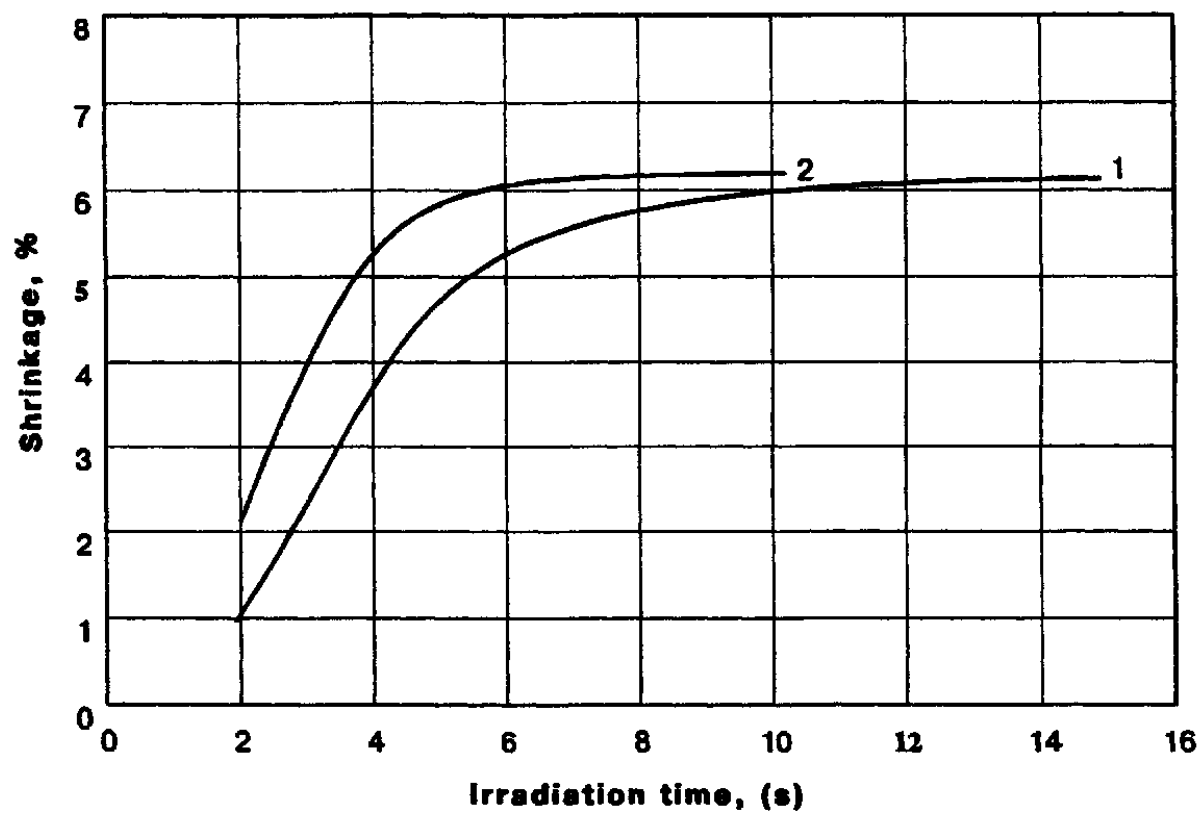

Fig.2. The correlation between the shrinkage and time of photocuring.

It should be mentioned that considerable shrinkage occurs after switching off the light. This is likely to be caused by a slow post-reaction as well as by reaction of shrinkage with respect to chemical conversion [9].

Figure 3, comparing shrinkage with viscosity for the glass adhesive formulation of 60 w.p. IF 491, IF 591 and IF 691 with 40 w.p. IBOA, reveals a striking result: the higher viscosity, the lower the shrinkage. Theoretically, it is to be expected that the higher the initial concentration of double 
bonds, corresponding to the highest number of double bonds being converted into polymeric single carbon-carbon-bonds, the higher the shrinkage. This would give e.g. IF 491 a low molecular weight, trifunctional oligomer theoretically a higher shrinkage than IF 591, or IF 691, being medium or high molecular weight difunctional oligomers. The difference in viscosity turns out to play a far greater role than difference in equivalent weight. There are two possible explanations for this phenomenon. The first one is that the differences in the rapidly increasing viscosities during polymerization makes it more difficult, in the case of a higher viscosity to relax to the most favorable conformation. The second one is that the lower the viscosity, the higher the mobility of the reacting species, which results in a more effective cross-linking, i.e. more of the available double bonds are actually used for cross-linking.

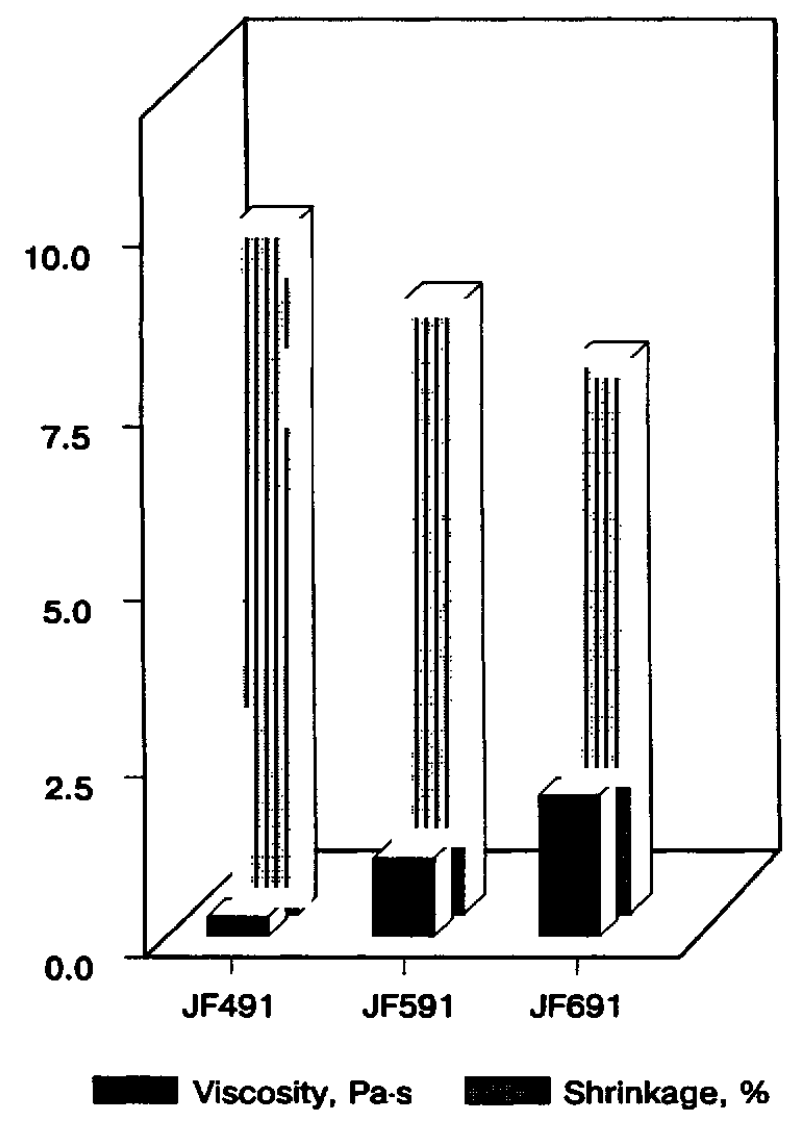

Fig.3. The correlation between the shrinkage and viscosity of glaeses adhesives.

\section{Adhesion}

Striking is the excellent adhesion of multifunctional acrylates HEOTMPTA, DPETPA, XPCM and of the formulation IF 491. This shows that again there seems to be a positive rather than negative correlation between polymerization shrinkage of oligomers and adhesion.

\section{Hardness}

More soft relief-carrying coating of the video disc offers less resistance to deformation by stress 
in the reflective mirror than hard coating. From this point of view the high functionalized monomers are preferred. With great advantage the additive of EAS- 637 oligomer can be used (see table 3).

Table 3

Properties of relief-forming compositions

\begin{tabular}{lllllc}
\hline Coating & $\begin{array}{l}\text { Mixture * } \\
\text { (w.p.) }\end{array}$ & $\begin{array}{l}\text { Viscosity } \\
\text { at } 25^{\circ} \mathrm{C} \\
(\mathrm{mPa})\end{array}$ & $\begin{array}{l}\text { Time of } \\
\text { photocuring } \\
\text { in inert atm.(s) }\end{array}$ & $\begin{array}{l}\text { Volume } \\
\text { shrinkage }\end{array}$ & $\begin{array}{l}\text { Hardness } \\
(\mathrm{MPa})\end{array}$ \\
\hline A & $\begin{array}{l}\text { 65 BDDA } \\
\text { 35 EAS-637 }\end{array}$ & 40 & 6 & 11.7 & 20.5 \\
B & \begin{tabular}{l}
$100 \mathrm{HDDA}$ \\
\hline
\end{tabular} & 6.7 & 6 & 12.6 & 11.0 \\
\hline
\end{tabular}

* 100 w.p. composition were mixed with 3 w.p. photoinitiator.

\section{Optical characteristics}

The increasing of the refractive index is essential for reproduction of the optical discs. The refractive index increases, if the molecule of the monomer contains halogens and aromatic rings. Among halogen-containing acrylic monomers with high refractive index TBNPACM, TBDPDCM, DBNPGDCM are the most interesting.

High birefringence index results in a worsening of the relief quality and in the noise level increasing. Birefringence of the polymers depends mainly from internal stresses in the cross-linked material. We have found that the replacement of (meth)acrylates by the corresponding carbonate (meth)acrylates gives extremely low birefringence index values.

Table 4 compares the properties of the whole photopolymer substrates of digital optical discs on the XPCM (A) [8], BPADM (B) basis, the formulation of DPETPA, IBOA and urethanemethacrylate $(C)$ [7].

Table 4

Properties of photopolymer substrates

\begin{tabular}{llllll}
\hline Mixture* & $\begin{array}{l}\text { Viscosity } \\
\text { at } 25^{\circ} \mathrm{C} \\
(\mathrm{mPa} . \mathrm{s})\end{array}$ & $\begin{array}{l}\text { Volume } \\
\text { shrinkage } \\
(\%)\end{array}$ & $\begin{array}{l}\text { Refractive } \\
\text { index }\end{array}$ & $\begin{array}{l}\text { Birefrin- } \\
\text { gence } \\
(\mathrm{nm} / \mathrm{mm})\end{array}$ & $\begin{array}{l}\text { Tensile } \\
\text { strength } \\
(\mathrm{MPa})\end{array}$ \\
\hline & & & & & \\
$\mathrm{A}$ & 258 & 5.5 & 1.52 & 2.8 & 73.0 \\
B & 1600 & 5.8 & 1.54 & 40 & 35.0 \\
$\mathrm{C}$ & 500 & 7.0 & 1.51 & 3.5 & 51.0 \\
\hline
\end{tabular}

* 100 w.p. of formulation were mixed with 3 w.p. of photoinitiator 


\section{Conclusion}

The widely used and new (meth)acrylate monomers were investigated. The particular properties of multifunctional carbonate methacrylates provide for their application in the manufacturing of optical discs. Among the oligomers for laminated glasses multifunctional urethane methacrylates are also the most preferable.

\section{References}

1. G. Bouwhuis, J.Braat, A.Huijser et al., Ed. Adam Hilger Ltd., Bristol and Boston (1985) p 280.

2. R.G.Gossink, Anew.Makromol.Chem., 145/146 (1986).

3. A.Toshihiro, N.Jutaka, U.Kankichi, Europ.Pat.Appl. No 0119525 (1984).

4. A.S.Rot, I.N.Zaks, Polimery, 36, 3 (1991) 101 (in Polish).

5. V.V.Shibanov, A.S.Rot et al., JNIPFIC, 35, 3 (1991) 231.

6. V.K.Grishchenko, A.P.Maslyuk, S.S.Gudzera, Liquid photopolymerizable compositions, Ed., Naukova Dumka, Kiev (1985) 208 (in Russian).

7. S.Ryoichi, M.Hiroacu, T.Tetsuo and W.Takeshi, Eorop.Pat.Appl. No 0156372 (1985).

8. A.S.Rot, I.N.Zaks, I.A.Dovgal et al., LKM, 5, 8 (1990) 13.

9. J.G.Kloosterboer, G.M.M.Van de Hei, R.G.Gossink, G.C.M.Dortant, Polym.Commun., 25 (1984) 322. 